\title{
Del alteractivismo al estallido social: acción juvenil colectiva y conectiva (20II y 20I9)
}

\author{
Juan Carlos Amador-Baquiro, Ph.D. ${ }^{a}$ \\ Universidad Distrital Francisco José de Caldas, Colombia
}

Germán Muñoz-González, Ph.D. ${ }^{b}$

UniMinuto, Colombia

\section{Resumen (analítico)}

En este artículo analizamos la participación de los jóvenes en los movimientos alteractivistas de 2011 y los estallidos sociales de 2019, quienes, al parecer, han sido protagonistas de nuevas formas de acción colectiva y conectiva. Desde el enfoque cualitativo de investigación y el método de análisis documental, se revisó un corpus de 61 documentos que comprenden literatura académica e información mediática sobre estos hechos. En los resultados se destacan: la redefinición ontológica y sociopolítica de la condición juvenil a partir de la acción colectiva; el tránsito de prácticas políticas antiglobalización a repertorios de acción colectiva más radicales que buscan resistir a la precariedad y la violencia; y acciones conectivas que transitan de estrategias de replicación a intercambios entre gestores de contenidos y audiencias, por medio de textos multimodales-performativos.

\section{Palabras clave}

Jóvenes, alteractivismo, estallido social, acción colectiva, acción conectiva.

\section{Thesauro}

Tesauro de Ciencias Sociales de la Unesco.

\section{Para citar este artículo}

Amador-Baquiro, J. C., \& Muñoz-González, G. (2021). Del alteractivismo al estallido social: acción juvenil colectiva y conectiva (2011 y 2019). Revista Latinoamericana de Ciencias Sociales, Niñez y Juventud, 19(1), 1-28.

https://dx.doi.org/10.11600/rlcsnj.19.1.4588

\section{Historial}

Recibido: 14.09.2020

Aceptado: 04.11.2020

Publicado: 17.12 .2020

\section{Información artículo}

El presente artículo hace parte de los resultados de la investigación titulada Memoria visual de jóvenes en el conflicto armado y la paz en Colombia (2002-2019), financiada por el Centro de Investigaciones y Desarrollo Científico de la Universidad Distrital Francisco José de Caldas (CIDC). Acta compromisoria $N^{\circ} 13$ de 2018 (inicio 1 de agosto de 2018, cierre 31 de marzo de 2020). Área: sociología. Subárea: socioloaía de la iuventud. 


\title{
From alteractivism to social outbreak: collective and connective youth action (2OII \& 2OI9)
}

\begin{abstract}
(analytical)
In this article we analyze the participation of young people in the alteractivist movements of 2011 and the social outbreaks of 2019, who, apparently, have been protagonists of new forms of collective and connective action. From the qualitative research approach and the documentary method, we check a corpus of 61 documents about academic literature and media information. The results show: the ontological and sociopolitical redefinition of the youth condition based on collective action; the transition from anti-globalization political practices to more radical repertoires of collective action that seek to resist precariousness and violence; and connective actions that go from replication strategies to exchanges between content managers and audiences, through multimodal-performative texts.
\end{abstract}

Keywords

Youth, alteractivism, social outbreak, collective action, connective action.

\section{Da alterativismo ao surto social: ação coletiva e conectiva da juventude (20II e 20I9)}

\section{Resumo (analítico)}

Neste artigo, analisamos a participação dos jovens nos movimentos alterativistas de 2011 e os surtos sociais de 2019, que, aparentemente, foram protagonistas de novas formas de ação coletiva e conectiva. A partir da abordagem qualitativa da pesquisa e do método de análise documental, foi revisado um corpus de 61 documentos que compõem a literatura acadêmica e informações midiáticas sobre esses eventos. Destacam-se os resultados: a redefinição ontológica e sociopolítica da condição juvenil a partir da ação coletiva; a transição das práticas políticas antiglobalização para repertórios mais radicais de ação coletiva que buscam resistir à precariedade e à violência; e ações conectivas que vão desde estratégias de replicação até trocas entre gestores de conteúdo e públicos, por meio de textos multimodal-performativos.

Palavras-chave

Juventude, alterativismo, surto social, ação coletiva, ação conectiva.

\section{Información autores}

[a] Posdoctor en Ciencias Sociales, Niñez y Juventud y Doctor en Educación. Profesor de la Universidad Distrital Francisco José de Caldas y del convenio Universidad de Manizales-Cinde. Integrante del grupo Jóvenes, culturas y poderes. (iD) 0000-0002-5575-1755. H5: 11. Correo electrónico: jcamadorb@udistrital.edu.co

[b] Investigador Emérito por reconocimiento de Colciencias (2018). Filósofo de la Universidad de San Buenaventura (Bogotá), Magíster en Semio-lingüística de l'Ehess (Paris). Doctor en Ciencias Sociales, Niñez y Juventud (Centro de Estudios Avanzados en Niñez y Juventud de la Universidad de Manizales-Cinde). Docente Universidad de Manizales, Universidad Distrital Francisco José de Caldas (Bogotá) y Uniminuto (Bogotá). Líder del grupo de investigación Jóvenes, Culturas y Poderes del Centro de Estudios Avanzados en Niñez y Juventud de la Universidad de Manizales y el Cinde. iD 0000-0002-4564-5828. H5: 26. Correo electrónico: gmunozg2012@gmail.com 


\section{Introducción}

esde la década de 1990, con el levantamiento zapatista en Chiapas, las mani-
festaciones contra la Organización Mundial del Comercio en Seattle y la promulgación del Foro Social Mundial, en las versiones de Porto Alegre, Bombay y Nairobi, se inició un nuevo ciclo de movimientos sociales. Este se caracterizó por su oposición a las medidas corporativas y antiderechos promovida por el neoliberalismo, así como por proponer globalizaciones alternativas orientadas por el eslogan «otros mundos son posibles». Esta ola de movimientos de carácter glocal, en los cuales las acciones locales se empezaron articular con proyectos de resistencia de otras regiones y países, en torno a problemáticas comunes (Santos, 2010), tales como la pobreza, la destrucción de la naturaleza y la represión contra comunidades originarias, mujeres, jóvenes y personas homosexuales y transgénero, se convirtió progresivamente en un proyecto altermundialista. Pleyers (2018) lo entiende como un movimiento que, a lo largo de las últimas tres décadas, se ha configurado en una propuesta alternativa al sistema económico mundial, que además busca ampliar espacios democráticos. En estos procesos de movilización de actores sociales precarizados y marginales, los jóvenes también han jugado un papel decisivo.

Desde inicios del 2000, tres hitos articulan el altermundialismo y los estallidos sociales con continuidades y transformaciones, incluyendo movilizaciones, revueltas e insurrecciones en varios países. El primer hito comprende las revueltas de Seattle (1999), Cancún (1999 y 2003), Praga (2000), Barcelona (2001 y 2003), Génova (2001) y Porto Alegre (2001 y 2005). Además de promover la idea de imaginar la vida de otra manera —más allá del capitalismo-, esta primera ola se constituyó progresivamente en un movimiento antisumisión, el cual cuestionó la naturalización de la desigualdad y la exclusión como patrones del modelo capitalista. El segundo hito develó la obsolescencia del orden económico mundial, presionó la modificación de poderes caducos de tipo nacional y local en muchos países y visibilizó las tecnologías digitales y las redes como mediaciones estratégicas para efectuar luchas de tipo social, político y cultural. Emergió en los movimientos de 2011-2012: Ocuppy Wall Street (Estados Unidos), los indignados del M-15 (España), las 
revueltas árabes de Túnez, Egipto, Libia, Siria, Yemen y Argelia —entre otros países del medio oriente-, así como los movimientos estudiantiles de Chile y Colombia. Por último, con los estallidos sociales de 2019, los cuales tuvieron como epicentro a Asia Oriental, Oriente Medio, Europa Occidental y América Latina, se amplió y consolidó la participación de los jóvenes, quienes asumieron que la radicalización de las medidas neoliberales (ahora iliberales y ultraconservadoras), no solo precariza a los trabajadores y se constituye en un sistema criminal que atenta contra la vida humana y no humana, sino que también pone en riesgo el futuro de las siguientes generaciones.

Este sintético recorrido muestra que durante la última década se han intensificado las protestas en varios países, como consecuencia de la profundización de medidas macroeconómicas orientadas por organismos internacionales, basadas en las leyes del mercado, la privatización de lo estatal, la mercantilización del bienestar social y la explotación de la naturaleza. También muestra que existe una preocupación creciente en diversos sectores de la sociedad por la reducción progresiva de espacios democráticos y de garantías para la defensa de los derechos humanos (Pleyers, 2018). Así mismo, plantea que, tanto en los procesos de movilización social de 2011 como en los de 2019, los jóvenes estudiantes han cumplido un rol protagónico en la mayoría de las movilizaciones, asumiendo la defensa de la educación pública secundaria y universitaria y, cada vez más, jóvenes marginales y precarizados exigiendo oportunidades para estudiar, trabajar y construir una vida digna.

Dichas protestas emplean formas convencionales de los movimientos sociales clásicos (Cabalín-Quijada, 2014) y elementos novedosos, especialmente en lo que refiere a la complementariedad entre la acción colectiva y la acción conectiva; se reconfigura así, no solo la participación y la cultura política juvenil, sino también los procesos de subjetivación de los jóvenes que hacen parte de estas experiencias.

Más allá del debate sobre los viejos, nuevos y novísimos movimientos sociales, esta perspectiva busca responder a una serie de interrogantes sobre el supuesto carácter efímero del uso de las tecnologías digitales en estos procesos, así como la aparente tendencia hacia el individualismo, la fragmentación y la falta de unidad de estas acciones en el sostenimiento de proyectos políticos (Morán \& Benedicto, 2008; Scherman \& Arriagada, 2010). Por otro lado, se trata de una mirada de la acción colectiva y conectiva juvenil que busca responder a inquietudes relacionadas con las paradojas que surgen de un grupo social que se interesa por defender la democracia y los derechos, a la vez que se distancia 
de procesos de participación formal, especialmente en lo que concierne a su desinterés en los procesos electorales.

La acción colectiva se ha entendido como el conjunto de procesos y prácticas efectuados por grupalidades y organizaciones sociales que buscan incidir en las pautas culturales, económicas y de sociabilidad, las cuales son ejecutadas de forma autoorganizada (Aguilera, 2014; Castells, 2003). Analizaremos algunos movimientos que, de manera explícita, incorporan en su repertorio de prácticas tanto formas de acción colectiva en el espacio físico como iniciativas conectivas en el espacio virtual. Aunque son diversos, se seleccionaron movimientos de países que se destacaron durante estos dos últimos hitos por el protagonismo de los jóvenes, la configuración de comunidades de sentido y el carácter situado de sus prácticas políticas, a partir de dos dimensiones: los contextos socioculturales amplios en los cuales los jóvenes se expresan y los procesos que hacen posible la constitución de agrupamientos en un proceso complejo no exento de tensiones. ${ }^{1}$

Por su parte, la acción conectiva refiere a movilizaciones a gran escala, sin liderazgos ni estructuras jerárquicas visibles, alimentadas por prácticas comunicativas mediadas por tecnologías digitales y herramientas de Internet, gestadas informalmente por ciudadanos identificados con la misma causa (Bennett \& Segerberg, 2011). Estas prácticas comunicativas se gestionan a partir de un lenguaje común y un modelo de coautoría que se introduce en los espacios digitales, el cual va dando forma a cierta inteligencia colectiva, de comunes conectados que devienen protagonistas.

En esta línea de reflexión, analizaremos tres aspectos. En primer lugar, luego de describir el papel de los jóvenes en estos dos hitos de protestas, analizaremos la relación entre la acción colectiva y las transformaciones ontológicas y sociopolíticas de la condición juvenil. Esta última, como se observará más adelante, se caracteriza por su abierta disputa con las instituciones, los grupos hegemónicos y la democracia formal, así como por una redefinición de la participación desde la multiplicidad y la diferencia. Por otro lado, además de develar cómo en el primer hito sobresale una postura antiglobalización que se enriquece desde prácticas comunicativas y performativas juveniles, se explicará cómo en los estallidos sociales de 2019 las acciones colectivas buscan responder a la extrema precariedad, el desencanto y la violencia procedente del Estado y los grupos de poder. Por último, se revisarán las acciones conectivas de ambos movimientos, destacando los

\footnotetext{
${ }^{1}$ Se utilizarán de manera conjunta los principales aportes de la teoría de movilización de recursos, la estructura de oportunidades políticas y de marcos identitarios como formas de abordar en su complejidad la acción colectiva juvenil (Aguilera, 2014).
} 
procesos de replicación y redifusión de la información digital en los alteractivistas de 2011, así como los intercambios entre gestores de contenidos y audiencias, por medio de textos multimodales, como una forma de expandir y esparcir las acciones colectivas tanto en los espacios digitales como sociales y físicos en los estallidos sociales de 2019.

\section{Método}

Teniendo en cuenta que la investigación tuvo como propósito comprender el despliegue de estas acciones de participación y comunicación por parte de jóvenes ubicados en algunos países de Asia Oriental y del Sur, Medio Oriente, Europa Occidental, Norteamérica y América Latina en estos dos hitos de protestas (2011 y 2019), tanto en su variabilidad como en sus continuidades, optamos por un enfoque de investigación cualitativa y una estrategia metodológica basada en el análisis documental. En relación con el enfoque, asumimos la investigación cualitativa como un campo constituido de diferentes perspectivas y orientaciones, las cuales se apoyan de diversas tradiciones epistemológicas, ontológicas y disciplinares, con sus correspondientes prácticas, métodos y procedimientos. El campo contiene distintos discursos, prácticas y actores sociales, y su objeto es la comprensión de la realidad social, así como el diseño e implementación de criterios y procedimientos que permitan producir conocimiento sobre esta en condiciones de legitimidad (Vasilachis, 2007). Además de ser una base epistémica fundamental para guiar estudios empíricos, el enfoque cualitativo también puede orientar investigaciones a partir de diversas fuentes de información y materiales con el fin de encontrar regularidades y discontinuidades en torno a hechos sociales. Una de estas posibilidades es la investigación documental.

De acuerdo con Galeano (2018), la investigación documental es una estrategia metodológica que se apoya en la construcción de archivos y corpus, los cuales, además de convertirse en los materiales centrales para realizar procesos de carácter interpretativo, hacen posible construir investigaciones de tipo teórico sobre hechos sociales, situados en coordenadas espacio-temporales específicas. A partir de la información adquirida en el corpus, se elaboran datos que se confrontan y triangulan con otro tipo de fuentes, entre ellas, las procedentes de otras investigaciones y hasta de otro tipo de archivos. Aunque en la literatura existen métodos valiosos (como el análisis de contenido), el análisis documental propone una serie de técnicas que fomentan la elaboración de notas y memos analíticos para identificar patrones, recurrencias, tendencias, convergencias y contradicciones, 
por medio del levantamiento de códigos, categorías y lecturas cruzadas de los documentos que conforman el corpus.

Como procedimiento, luego de construir el objeto de investigación y definir los objetivos del estudio, desarrollamos cuatro momentos. En el primero, se adelantó el rastreo, inventario y muestreo de documentos existentes y disponibles. Específicamente, se llevó cabo una revisión inicial de 102 documentos, entre ellos artículos científicos procedentes de las bases de datos Science Direct, Redalyc, Latindex, DOAJ, Dialnet y Clacso (las cuales se destacan en el campo de las ciencias sociales por divulgar permanentemente reportes de investigación sobre movimientos sociales), así como notas de prensa, crónicas y columnas de opinión provenientes de portales que cubrieron los acontecimientos de 2019. ${ }^{2}$ En el segundo momento se efectuó la clasificación y selección de los documentos conforme a los tópicos (acción colectiva y acción conectiva), los escenarios sociales y geopolíticos, el periodo (2011 y 2019), la descripción de los acontecimientos y el tipo de análisis propuesto. Luego de proceder con los filtros semánticos, se seleccionaron 61 fuentes escritas. Por cada uno de los documentos seleccionados se diseñó una ficha analítica que incluyó los datos descriptivos de la macroestructura, la interpretación del enfoque empleado por el autor y los aspectos sociohistóricos de los dos hitos. En el tercer momento se realizó una sistematización de datos por medio de una matriz descriptiva que incluyó la información textual, los códigos y los campos semánticos. Y en el cuarto momento se procedió con el análisis documental integral, así como con la valoración y la interpretación de lo hallado. En relación con este último momento, es importante mencionar que se utilizó una matriz de análisis en la que se ubicaron los campos semánticos identificados en el momento anterior, los cuales permitieron reconocer las categorías emergentes. Luego de determinar las categorías, en la misma matriz fueron identificadas las líneas teóricas generales con el fin de efectuar la triangulación entre los datos, los conceptos y nuestras propias interpretaciones (Galeano, 2018).

2 Si bien se analizó un fenómeno de alcance global que evidencia aspectos comunes y particularidades en diferentes lugares del mundo, se privilegió la revisión documental en idioma español e inglés, dado que los reportes de investigación utilizan como referente internacional al menos dos idiomas. Según Thomson Reuters, el 79\% de los investigadores a escala mundial publican artículos en inglés. Esto hace que aumente exponencialmente su alcance y visibilidad (Torres, 2017). 


\section{Resultados}

\section{Jóvenes en los movimientos alteractivistas desplegados en 2011}

Según Juris et al. (2012), el alteractivismo, al que prefieren llamar novísimos movimientos sociales, contiene cinco características principales: un énfasis en el antiglobalismo y la transnacionalidad, así como su articulación con los contextos locales; el uso de las tecnologías de la información y la comunicación digitales; la articulación de las demandas económicas y las relacionadas con la identidad; el desarrollo de formas innovadoras de acción, especialmente de orden simbólico-performativo; y la creación de nuevas formas de organización, las cuales involucran diversas tradiciones y organizaciones bajo un marco compartido. Mientras que los clásicos movimientos sociales aluden a los procesos organizativos de trabajadores (desde finales del siglo XIX y hasta la primera mitad del siglo XX), los cuales se orientaron hacia demandas como la democratización y el derecho al voto, ${ }^{3}$ los nuevos movimientos sociales — surgidos desde la década de 1950 - reafirmaron como objeto de lucha la identidad, la diferencia y el reconocimiento.

Por su parte, los novísimos movimientos sociales se asocian con una nueva forma de ciudadanía en la sociedad global, la cual tiene como protagonistas a los jóvenes conectados a las pantallas, quienes, a la vez, hacen presencia de manera activa en las manifestaciones callejeras, las acampadas y las asambleas. Se trata de la emergencia de una sociedad global en resistencia que, no solo actúa conforme a las coyunturas - desde la protesta y la rebeldía-, sino que configura un modo de pensamiento orientado hacia la construcción de proyectos alternativos frente a la globalización hegemónica, desde la diversidad y la diferencia. Por esta razón, además de las cinco características expuestas anteriormente, estos movimientos logran movilizaciones políticas con diversos grupos de la sociedad, propician escenarios de encuentro e interacción de tipo intergeneracional e intersectorial, se constituyen en fuerzas culturales que dan voz a los marginales y producen otros modos de sociabilidad (Pleyers, 2018).

En las cuatro experiencias de acción colectiva y conectiva que se mencionan a continuación fueron protagonistas los jóvenes. El movimiento Occupy Wall Street surgió en el marco de la crisis económica de 2008-2011, la cual dejó a muchos países en situación

\footnotetext{
${ }^{3}$ Algunos ejemplos de estos movimientos son la comuna de París, la revolución rusa en 1917 y el movimiento a favor de la reforma universitaria en Córdoba (Argentina) en 1918 (Tarrow, 2011).
} 
de pobreza y desempleo. Dentro de los objetivos del movimiento se destacaron: ocupar permanentemente el distrito financiero de Nueva York, hacer explícitos los intereses oligopólicos de las corporaciones, evidenciar los efectos del sistema financiero en la desigualdad estructural y exigir al Gobierno la conformación de una comisión presidencial encargada de poner fin a las políticas que legitiman la concentración de capital con el apoyo de organismos internacionales (Chomsky, 2012; Tarrow, 2011). El movimiento tuvo varios críticos y detractores, pero se convirtió en viral luego de que personalidades como el filósofo Noam Chomsky, el activista Michael Moore, el escritor Juan Gelman y la artista Yoko Ono declararan públicamente su apoyo al movimiento (Enríquez-Román, 2018).

Desde el 17 de septiembre, día en el que se inició la ocupación, decenas de jóvenes ya acumulaban meses de trabajo organizativo, específicamente profesionales desempleados, quienes se encontraban inmersos en un mercado laboral con pocas posibilidades de movilidad social (Chomsky, 2012; Tarrow, 2011). La participación y el activismo juvenil se hizo notable por medio de AdBusters, proyecto que utiliza la publicidad como medio de comunicación de ideas críticas. Aunque sus integrantes tienen perfiles muy diversos (entre ellos, escritores, periodistas y adultos comprometidos con el anticonsumismo), sus principales impulsores son activistas jóvenes, quienes ejercen roles de estudiantes o trabajadores, aun cuando muchos son desempleados. De acuerdo con Juris (2012) y Juris y Razsa (2012), muchos jóvenes estadounidenses, incluso migrantes, fueron capaces de convocar sectores amplios de ciudadanos de distintas edades y sin filiaciones políticas definidas.

En segundo lugar, el movimiento 15-M, también llamado «de los indignados», se manifiesta durante 2011 en España. Alrededor de lemas, como «No somos marionetas en manos de políticos y banqueros» $\mathrm{y}$ «democracia real iya!», entre otros, los activistas que conformaron la base de este movimiento organizaron progresivamente colectivos temáticos de discusión que sentaron las bases para construir un discurso común. Para FernándezPlanells et al. (2013), el 15-M es un movimiento que innovó al complementar las acciones tecnopolíticas y las acciones de movilización en las calles. Actividades como las acampadas, las asambleas y los trabajos de las plataformas y colectivos se caracterizaron por asumir como criterios de acción social la horizontalidad, la inteligencia colectiva, la no violencia, el uso de las tecnologías digitales y el rechazo a los personalismos.

Los jóvenes indignados cumplieron roles fundamentales en los diversos procesos de movilización. El perfil predominante del participante de una acampada era un «chico o chica, estudiante, de entre 18 y 25 años (...). Los participantes en el 15-M reclamaban el espacio público como lugar para el debate, la organización y la participación y acción 
política» (Fernández-Planells et al., 2013, p. 125). Los grupos de trabajo, organizados por grandes áreas temáticas (sanidad, educación, derechos humanos, entre otras), decidían determinadas acciones en las comisiones y, posteriormente, llevaban las propuestas a la asamblea general. Así mismo, se trata de un movimiento informacional y organizado en torno a redes flexibles y descentralizadas, sin portavoces oficiales (Antebi \& Sánchez, 2012). Además de protestas apoyadas en el arte-activismo en la calle, los jóvenes alteractivistas también emplearon estrategias trending topic, las cuales incluyen videos virales $\mathrm{y}$ «escraches».

En tercer lugar, las revueltas árabes se iniciaron en Túnez, en diciembre de 2010, a partir de las intensas manifestaciones originadas por la inmolación de Mohamed Bouazizi, un joven vendedor ambulante que decidió acabar con su vida luego de que la policía confiscara su puesto de frutas y lo humillara. La escalada de protestas surgida tras este hecho, así como los consecuentes actos de represión policial, trajeron consigo la dimisión de Ben Alí en los primeros días de enero de 2011. A partir de esta conquista del movimiento tunecino, surgieron nuevas movilizaciones: en Egipto, contra Hosni Mubarack; en Libia, contra Muamar Gadafi; en Siria, contra Bashar Al Assad; en Yemen, contra Ali Abdullah Saleh; y en Argelia, contra Abdelaziz Buteflika. Además de lo ocurrido en Túnez, en Egipto fue derrocado Mubarak y en Libia Gadafi fue ejecutado tras la intervención de la Otán meses después. En Yemen, dimitió Abdullah Saleh, y en Siria se dio inicio a una guerra civil que se extiende hasta la actualidad (Soengas-Pérez \& Assif, 2017). De acuerdo con Willis (como es citado por Pérez-Beltrán, 2012), la mayoría de las sociedades árabes alcanzaron tal nivel de hartazgo que terminaron por salir a las calles a rebelarse contra sus respectivos gobiernos tiránicos con el fin de reivindicar tres asuntos primordiales: libertades democráticas, mejoras del nivel de vida y el fin de la corrupción y de la impunidad.

Pérez-Beltrán (2012) señala que los jóvenes fueron protagonistas de este movimiento no solo por su capacidad de convocatoria y expresión creativa en las calles y en las redes, sino también porque es el grupo social que acumula mayor frustración en la estructura social, dado que no puede satisfacer gran parte de sus necesidades básicas, especialmente los mayores de 25 años, quienes constituyen la mitad de la población árabe en la región. ${ }^{4}$ Por otro lado, los jóvenes con una mayor formación académica también estaban desocupados, pues el zo por ciento de los desempleados de Marruecos y el 21.6 por ciento de los de Túnez contaban con formación universitaria (Achy, 2010). En otras palabras, los jóvenes

\footnotetext{
${ }^{4}$ Los jóvenes también sufren el desempleo, así: el $72 \%$ de los desempleados tunecinos, el $75 \%$ de los argelinos, el $62 \%$ de los marroquíes son menores de zo años (Achy, 2010).
} 
que impulsaron estos movimientos eran desempleados, no tenían recursos, no tenían esperanza de un mejor futuro y muchos vivían en regímenes represivos, condicionados por marcados valores patriarcales, racistas y clasistas (Valdettaro, 2012).

Por último, los movimientos estudiantiles en Chile y Colombia también hicieron parte de este hito alteractivista. En el caso de Chile, luego de revueltas estudiantiles como la de los «Pingüinos» en 2006 y de avances en procesos de organización universitaria a lo largo de la primera década del 2000, durante 2011 se llevaron a cabo varias manifestaciones por parte de estudiantes universitarios y de secundaria. Además de la privatización del sistema educativo, la precarización del empleo docente y el debilitamiento de la educación pública desde los tiempos de la dictadura de Pinochet (Aguilera, 2014), otras causas que motivaron las movilizaciones de 2011 fueron el desfinanciamiento de la educación universitaria, el intento de vender parte de la Universidad Central de Chile a un conglomerado económico y dilaciones con la gestión de la tarjeta nacional estudiantil. De acuerdo con Bellei y Cabalín (2013), los estudiantes transmitieron mensajes contundentes que sintetizaban muy bien los principales problemas del sistema educativo de Chile: desigualdad, mala calidad, segregación y endeudamiento.

En Colombia, el movimiento estudiantil de 2011 —representado en la Mesa Amplia Nacional Estudiantil- comprende un conjunto de manifestaciones y expresiones adelantadas por estudiantes de instituciones de Educación Superior desde abril hasta noviembre, a raíz del proyecto de reforma a la Educación Superior impulsado por el Gobierno de Colombia. La reforma buscaba dar vía libre a la implementación de universidades con ánimo de lucro, racionalizar los recursos de las universidades estatales, implementar un modelo de venta de servicios para que las universidades estatales compitieran por recursos públicos, así como limitar los recursos destinados a las instituciones de educación superior estatales de manera temporal. Luego de varias asambleas, marchas y expresiones mediáticas a lo largo de cinco meses, y luego de la declaratoria de paro nacional universitario en septiembre, el Gobierno Nacional retiró el proyecto de reforma del Congreso el 10 de noviembre (Cruz-Rodríguez, 2012).

En cuanto al movimiento estudiantil de Chile, se trata no solo de jóvenes vinculados a estos procesos organizativos sino de formas de politización juvenil que se inscriben en una perspectiva cultural e intergeneracional. De acuerdo con Aguilera (2014), el movimiento estudiantil de 2011 comprende un conjunto de acciones que reconfigura la cultura política juvenil, situación que se puede evidenciar en discursos y prácticas diferenciados respecto al campo político institucional, así como sus procedimientos, actores y estrategias 
(Aguilera, 2014; Cárdenas, 2014). Por último, el movimiento estudiantil en Colombia evidenció que los jóvenes fueron protagonistas en las distintas acciones de movilización, las cuales convocaron a otros grupos y sectores de la sociedad (Cruz-Rodríguez, 2012). Durante las movilizaciones se desplegó un amplio repertorio de acciones, entre ellas: foros y debates públicos; acciones de tipo carnavalesco (abrazos en los campus universitarios, pupitrazos, puestas en escena en espacios públicos y besatones); marchas de antorchas; tomas de instituciones educativas; así como bloqueos de vías y tropeles. Además de este tipo de movilizaciones callejeras, los jóvenes produjeron contenidos digitales que se publicaron en redes sociales y otros sitios web. Algunos - como infografías, fotografías y videos- llegaron a diversos públicos a partir de lenguajes sencillos, satíricos y críticos (García, 2012).

\section{De Hong Kong a Chile: jóvenes en los estallidos sociales de 2019}

Durante 2019, especialmente a lo largo del segundo semestre del año, se produjo una explosión de protestas en muchos lugares del planeta, entre las que se destacan Hong Kong, Pakistán, Iraq, Líbano, Irán, Francia, Reino Unido, Cataluña, Chile y Colombia, entre otros países. Aunque aún no existe una conceptualización académica sobre la expresión estallidos sociales, a partir de la experiencia de Chile y Colombia, estos se entienden como un conjunto de acciones colectivas, procedentes de distintos sectores marginales, que buscan agrietar las estructuras culturales y las políticas dominantes (Aguilar-Forero, 2020), como consecuencia del abuso de poder ejercido por élites políticas y económicas. Estas, a partir de medidas que vulneran los derechos humanos por medio de las privatizaciones, la desregulación de la economía y la apropiación de los bienes comunes (Zibechi, 2010), precarizan progresivamente las condiciones de vida de los ciudadanos e imponen el desencanto como un imaginario que naturaliza la resignación y la zozobra.

En el caso de Asia Oriental y del Sur, las primeras protestas estallaron en Hong Kong, desde el mes de junio, a raíz de un proyecto de ley que autorizaría la extradición a China de personas que supuestamente representan peligro para la sociedad. Frente a esta medida, los convocantes a las movilizaciones argumentaron que se trata de un claro ataque a los derechos de los ciudadanos, que evidencia el control de China sobre la excolonia británica (Ives \& Li, 2019). Por su parte, en Pakistán las protestas antigubernamentales se iniciaron el 27 de octubre con una marcha multitudinaria de la ciudad de Carachi hacia la capital, tras los cuestionamientos al primer ministro, Imran Khan, por supuesto fraude electoral y una serie de medidas económicas que llevaron al Gobierno a solicitar un 
paquete de ayudas del Fondo Monetario Internacional condicionadas a la disciplina fiscal y la reducción del déficit (Sajid, 2019).

Los jóvenes de Hong Kong (mayoritariamente estudiantes universitarios y de secundaria), suelen usar camisetas negras, cascos y gafas de seguridad, así como emplear mensajes cifrados en las redes. Asumen que el problema va más allá de la ley que permitiría la extradición a China continental, pues se trata de una generación que, aunque no vivió directamente el régimen del Gobierno británico, ha crecido en una época de temores crecientes alimentados por la injerencia del Partido Comunista de China en los modos de vida de las personas en Hong Kong. Así mismo, denuncian una serie de medidas antidemocráticas por parte de Carrie Lam, Jefe Ejecutiva de la ciudad; entre ellas, la destitución de legisladores de oposición, la desaparición de personas de Hong Kong que estaban en custodia en China y la dificultad cada vez mayor para obtener empleo y vivienda en un espacio urbano de creciente desigualdad (Ives \& Li, 2019).

En Medio Oriente, las protestas comenzaron el 1 de octubre cuando miles de iraquíes, predominantemente shiítas - situados en el sur del país_, se lanzaron a las calles a manifestarse en contra de la corrupción, las alzas en los servicios públicos, la falta de empleo, así como la injerencia de Irán en su Gobierno. La represión cobró la vida de al menos 400 manifestantes (Amnistía Internacional, 2019), en su mayoría jóvenes, lo que llevó a que el primer ministro, Adel Abdul Mahdi, dimitiera ante el parlamento, el cual aceptó su renuncia. En el Líbano, las movilizaciones comenzaron el 17 de octubre, una vez el Gobierno anunció un nuevo impuesto para los servicios de mensajería gratuita por Internet. Aunque el Gobierno retiró la medida, las protestas no se detuvieron y se convirtieron en un movimiento amplio contra la corrupción y la incapacidad de las élites políticas para garantizar servicios sociales básicos. Esto llevó a que el primer ministro, Saad Hariri (sunnita), dimitiera el 29 de octubre («Protestas en Líbano», 2019). En Irán, el aumento del precio del combustible hizo estallar las protestas desde el 15 de noviembre. Como respuesta, las fuerzas represivas de Hasán Rouhaní llevaron al asesinato de al menos 143 manifestantes, la mayoría jóvenes (Amnistía Internacional, 2019).

En el caso de Iraq, las movilizaciones han estado lideradas por jóvenes desempleados («Decenas de muertos», 2020). Algunas de las situaciones que han desatado el descontento son la escasez de servicios médicos y educativos, así como la falta de acceso a agua potable y luz eléctrica. En el Líbano los jóvenes también han sido el centro de las protestas. Una de las acciones que han adelantado es la presencia masiva en la bahía de Zaitunay, un lugar público que fue privatizado y que se convirtió en sitio exclusivo de la clase alta. 
Quienes acuden a las manifestaciones consideran que este lugar es símbolo de una clase política odiada a la que califican de prepotente y corrupta. Por su parte, la fuerza de la movilización en Irán proviene de jóvenes universitarios de cinco ciudades. Las protestas en varias ciudades, dirigidas contra el régimen de la República Islámica, busca la dimisión del Gobierno, así como del más importante líder político-religioso del país, el Ayatolá Jamenei. De acuerdo con BBC News (Paúl, 2019), se trata de una clara radicalización del movimiento social, el cual se identifica con lemas como « No tengan miedo! Estamos juntos» $\mathrm{y}$ «Témannos, porque estamos juntos!».

Cataluña también fue escenario de importantes movilizaciones en octubre, asociadas con el rechazo a la condena judicial contra algunos líderes de esta comunidad autónoma acusados de sedición por promover el referendo y la independencia de Cataluña en 2017. Una de las expresiones más relevantes de este proceso fueron las llamadas «marchas por la libertad», las cuales partieron de Berga, Casteldefels, Gerona, Tarragona y Vich hacia Barcelona, suceso que se articuló con la huelga general catalana del 18 de octubre. Se calcula que cerca de 500 ooo jóvenes se sumaron a las protestas, entre independentistas revolucionarios y ácratas. A estos grupos se han sumado otros miles de jóvenes, sin filiación política o partidista alguna, dispuestos a efectuar marchas pacíficas o emplear la violencia de ser necesario. De acuerdo con Feixa (como es citado por Martín, 2019, § 3), las intenciones de estos grupos van más allá del independentismo: «Vemos una pluralidad de perfiles, entre los que hay una base de clase media de estudiantes que ven su futuro en cuestión (...). Es la primera vez desde la Transición que los jóvenes ven que van a vivir peor que sus padres. Una Cataluña independiente es para ellos la única utopía concreta». ${ }^{5}$

En América Latina se destacan los estallidos sociales de Chile, Ecuador y Colombia. En el caso de Chile, a raíz del aumento en el precio del tiquete del metro a principios de octubre, se produjeron las primeras protestas en Santiago. A pesar de que el presidente Piñera anuló la medida, las protestas se intensificaron y se convirtieron en lo que varios

\footnotetext{
5 Otro foco de movilizaciones en Europa ha sido Francia, por parte de los llamados «chalecos amarillos». A diferencia de lo ocurrido en otros países, estas revueltas se caracterizaron por la presencia masiva de manifestantes de clase media y algunos grupos de sectores rurales, quienes además de oponerse al proyecto de reforma pensional, exigieron justicia social y mejoras en su poder adquisitivo. El Gobierno del presidente Macron efectuó cerca de 11 ooo detenciones (Rojas, 2019). La participación de los jóvenes en los estallidos sociales de Francia cuenta con, al menos, dos grandes grupos de participantes. Por un lado, estudiantes universitarios que protestan por lo que denominan precariedad universitaria, luego de que el 8 de noviembre el joven Anas Kournif, un estudiante migrante, intentara inmolarse, prendiendo fuego a su cuerpo frente a la sede de Crous en Lyon, debido a que le retiraron su beca de estudios. Por otro lado, miles de jóvenes y estudiantes de diversas procedencias se unieron a las manifestaciones convocadas por los chalecos amarillos.
} 
analistas han llamado «estallido social». A partir de lemas como «Chile despertó» $\mathrm{y}$ «No son 30 pesos, son 30 años», las manifestaciones — las cuales llegaron hasta febrero de 2020-, también se desarrollaron en ciudades como el gran Valparaíso, Arica, Iquique, Antofagasta, Valdivia y Punta Arenas. Los reclamos crecieron por el alto costo de vida y los servicios de salud, el bajo nivel de las pensiones y un profundo rechazo a la clase política y los bancos (Paúl, 2019). En febrero de 2020 se confirmaron 32 civiles fallecidos como consecuencia de las revueltas y las brutales acciones policiales, 3400 personas hospitalizadas producto de los enfrentamientos (entre ellos más de 400 con pérdida de sus ojos por perdigones) y cerca de 8000 detenidos («Las cifras que dejó», 2020).

La encuesta Participación, jóvenes y consumo de medios (Scherman et al., 2020), informa que el $61 \%$ de los jóvenes de 18 a 29 años hizo parte de alguna manifestación en la vía pública, cifra que casi duplica el $32 \%$ de los participantes de las protestas de 2011 . El $64 \%$ de esta población utilizó redes sociales y otras aplicaciones para manifestarse; el $43 \%$ se mostró interesado en noticias políticas (frente a $24 \%$ en 2018) y el $45 \%$ manifestó estar interesado en conversar de política con amigos y familiares (en comparación al zo \% de 2018). Respecto a las movilizaciones que comenzaron en octubre, la participación fue amplia: $52 \%$ fue parte de cacerolazos; $50 \%$ difundió información en redes sociales; $49 \%$ asistió a concentraciones o marchas; y $15 \%$ protegió su lugar de estudio o trabajo. Por último, el estudio indica la fuerte relación entre las manifestaciones en la vía pública y la emisión de opinión en redes sociales, las cuales no son actividades paralelas, sino que forman parte de un mismo circuito.

Finalmente, en Colombia, luego de que varios sectores sociales —entre ellos indígenas, sindicatos y estudiantes - declararan un paro nacional a partir del 21 de noviembre, se inició una escalada de movilizaciones, concentraciones y cacerolazos en las principales ciudades del país, las cuales llegaron hasta el mes de diciembre. Además de la llamada Ley de financiamiento, liderada por el Gobierno de Iván Duque, la cual beneficiaría a los bancos y a los grandes empresarios, así como el anuncio de una reforma pensional y laboral, los manifestantes protestaron por el asesinato de ocho niños en un operativo militar en el departamento del Caquetá, la necesidad de implementar el acuerdo de paz con la exguerrilla de las Farc, garantías para defender la vida de líderes sociales, el aseguramiento de servicios básicos a poblaciones históricamente abandonadas por el Estado y la exigencia de educación gratuita y de calidad. Mientras que el presidente Duque se comprometió con un diálogo nacional como respuesta al descontento generalizado, se evidenciaron acciones represivas por parte del Escuadrón Móvil Antidisturbios, se retuvo 
ilegalmente a cientos de manifestantes y se registraron cerca de 200 heridos en varias manifestaciones. Dentro de estas acciones represivas, fue asesinado el estudiante de educación media Dylan Cruz por uno de los integrantes de este escuadrón, situación que profundizó el descontento e impulsó nuevas acciones (Manetto et al., 2019).

Miles de jóvenes universitarios protestaron para exigir el aumento de la base presupuestal de la educación pública; se sumaron jóvenes sin acceso a estudio ni trabajo, quienes consideran que no hay garantías para ser joven en el país. Más allá de exigir la dimisión del presidente Duque, los jóvenes participantes de las protestas exigieron garantías para trabajar, estudiar y vivir plenamente su juventud, sin que el establecimiento emplee estrategias de estigmatización y criminalización contra ellos. De acuerdo con Reyes (como es citada por Manetto, 2019), se trata de una generación comprometida con la lucha en las calles, unidos por el desencanto, el rechazo a la clase política y un profundo malestar frente al Gobierno. Por esta razón, perciben al presidente Duque, hijo de un exministro, en la otra orilla, como un representante de una generación de privilegios.

\section{Discusión}

De acuerdo con lo expuesto, y en sintonía con Aguilera (2014), Castells (2003) y Melucci (1999), los movimientos sociales comprenden procesos y prácticas efectuados por grupalidades y organizaciones sociales que buscan incidir en las pautas culturales, económicas y de sociabilidad, las cuales son ejecutadas de forma autoorganizada. De esta manera, al analizar el devenir de los movimientos altermundialistas de $2011 \mathrm{y}$ de aquellos que han hecho parte de los estallidos sociales de 2019, se puede afirmar que se requiere profundizar en el carácter situado de las prácticas políticas juveniles en una doble dimensión. Por un lado, desde los contextos socioculturales amplios en los cuales los jóvenes se desarrollan y expresan. Y, por otro, a partir de los procesos que hacen posible la constitución de agrupamientos en medio de la complejidad no exenta de tensiones. Estos aspectos coinciden con los elementos configuradores de la acción colectiva y conectiva.

\section{Acción colectiva y condición juvenil contemporánea}

En los dos hitos descritos, las prácticas sociales constitutivas de la condición juvenil contemporánea se definen en oposición a lo establecido, adoptando líneas de fuga y de invención. Sus formas de agencia generan sospecha, enrarecen el mundo, inventan tramas y 
no buscan mantener los privilegios históricamente constituidos, sino promover la creación, la resistencia, la transformación social y lo constituyente. Este tipo de acciones, además, en muchas ocasiones están mediadas por la inconformidad, el desacuerdo y la indignación, comprendidos como motores de un deseo transformador que lucha contra las opresiones, y que desata anomalías e impugna lo naturalizado y lo establecido. En suma, estas acciones colectivas emergentes son prácticas de transgresión que posibilitan pensar de otra manera la condición juvenil en el mundo de hoy.

En consecuencia, requerimos una ontología antiesencialista que reconozca el carácter múltiple, heterogéneo, dinámico, relacional, cambiante, nómada, frágil, transitorio y contradictorio de las identidades sujetas a procesos de construcción y reconstrucción continua. La condición juvenil contemporánea se articula en torno a nuevas temporalidades; a tiempos, ya no cronológicos (secuenciales, etarios, psicobiológicos), sino socioculturales y determinados por las condiciones de vida, contextos y marcos de posibilidad diferenciados. Se trata ahora de un devenir joven, de un acontecer particular, de trayectorias vitales difíciles de predecir, itinerantes, no-coherentes, con ritmos y rumbos diversos, cambiantes y difusos; con pasados obsoletos, presentes eternos y futuros azarosos e inciertos (Aguilar-Forero, 202O; Juris et al., 2012). Estas identidades colectivas emergentes, distintas a las de clase y agrupadas en este caso bajo los apelativos de alteractivismo y estallidos sociales, «además de ampliar el ámbito de las luchas sociales, trajeron consigo nuevas concepciones de vida y de dignidad humana, nuevos universos simbólicos, nuevas cosmogonías, gnoseologías y hasta ontologías» (Santos, 2010, p. 6o).

Las acciones colectivas desplegadas en el marco de los movimientos alteractivistas y de los llamados estallidos sociales de 2019, entendidas como formas de agencia y congregación alrededor de propósitos o motivaciones compartidas que articulan política, cultura y vida cotidiana (Amador \& Muñoz, 2018), pasan por relaciones expresivas y vínculos emocionales. Así mismo, hacen énfasis en la acción cotidiana y local, son más modestas en cuanto a las luchas y resultados esperados que el tradicional movimiento social obrero, se agrupan bajo referentes estéticos y simbólico-culturales, crean y experimentan formas diferentes de relaciones sociales y cuentan con una variable mediadora crucial: la identidad colectiva. Se trata entonces de una acción que no se fundamenta en la unidad, ni en la totalidad, sino en la potencia latente de la multiplicidad, de las singularidades, de la gran heterogeneidad posible (Lazzarato, 2006) en el estar juntos y estar en contra.

$\mathrm{Al}$ respecto, Hardt y Negri (2004) afirman que las estrategias de resistencia, las cuales en determinados momentos históricos se han revelado como más eficaces, han adoptado 
la misma forma que los modelos dominantes de producción económica y social. Por esta razón, la disputa en la red se convierte en fundamental para las formas de acción colectiva juvenil del mundo de hoy, que depende de la creatividad, la información, la comunicación y la cooperación autoorganizada de singularidades plurales. En este marco, en términos de Santos (2010), a la globalización hegemónica promovida por los grupos sociales dominantes se le contrapone una globalización contra-hegemónica propiciada por grupos subordinados que, por lo general, hacen un uso contra-hegemónico de discursos (como el de los derechos humanos) o tecnologías de acción a distancia (como las TIC digitales) fundamentales en la reproducción del sistema imperante.

En lo que refiere a los dos hitos descritos al inicio, de acuerdo con Pleyers (2018), se puede afirmar que las protestas y estallidos sociales ocurridos durante la última década, además de distinguirse por su carácter heterogéneo, se extienden por los lugares más disímiles, con tres contenidos comunes: a) el contexto de desaceleración económica que ha sentenciado en su asamblea el Fondo Monetario Internacional; b) la politización de la lucha contra la desigualdad (ya no es solo un problema social o económico, sino directamente político); y, c) una pronunciada caída en los niveles de identificación con los partidos políticos y con instituciones centrales como los gobiernos o los parlamentos. En suma, lo juvenil en las sociedades neoliberales, las cuales están atravesadas por la precariedad y la violencia institucionalizada, es una condición ontológica, social y política que implica la vinculación del sujeto joven a las acciones colectivas de resistencia y reexistencia por la justicia social, ambiental y cognitiva.

En este contexto, los jóvenes, que poco tienen que perder dado que se les ha convertido en sujetos precarizados, sin horizontes de futuro, en estados penales fuertemente represivos, continúan tomando la iniciativa para construir otros mundos posibles. Se trata de jóvenes que ya perdieron incluso el miedo, que no han mostrado interés en formas de organización tradicionales, del estilo de los partidos políticos o los viejos movimientos sociales, tal como ocurrió con los indignados del $15 \mathrm{M}$ y los activistas de las revueltas árabes del primer hito. Así como ellos, infinidad de colectivos juveniles, más o menos anónimos, sin pedir permiso, producen cambios sociales que mueven pensamiento, valores y formas estéticas. Algunas de sus principales características son: sin miedo; mediante formas-otras de lucha estratégica; sin liderazgos ni jefaturas; trascendiendo los partidos políticos y las polarizaciones clásicas; en defensa de la justicia social y ambiental, los derechos y las libertades de las diversidades; en tono artístico, festivo y creativo; y en busca 
de cambios radicales y duraderos. Estos elementos fueron comunes en las movilizaciones estudiantiles de Chile y Colombia del primer hito.

Otro aspecto que explica la presencia protagónica de los jóvenes en estos movimientos es lo que Feixa (como es citado por Martín, 2019) y Valenzuela (2015) han llamado «vidas al límite». Se trata de vidas que expresan el abandono y la indolencia de los poderes dominantes que desarrollan estrategias de control basadas en la biopolítica, y que se encuentran tanto en las políticas de población como en el conjunto de procesos y dispositivos a través de los cuales se ejerce poder y control sobre los cuerpos y vidas de las personas, tal como lo denunciaron los jóvenes de Hong Kong, Líbano, Irán, Chile y Colombia en el segundo hito. En esta línea de reflexión, Mbembe (2011) propone la necropolítica, entendida como políticas diferenciadas de poder soberano, inscritas en ordenamientos coloniales y desiguales que establecen qué grupos y personas son prescindibles y deben morir, así como quienes merecen vivir.

En concreto, vemos que miles de jóvenes que participan de estas acciones buscan responder de manera radical a la violencia institucional naturalizada en varios países. Buscan que la sociedad reconozca estas formas de violencia en escenarios sociales más amplios, los cuales incluyen procesos de precarización económica y social, la configuración de grupos, sectores o identidades juveniles desacreditadas, la fractura de los marcos axiológicos junto al descrédito de las instituciones, la construcción de cuerpos-territorios juveniles como ámbitos privilegiados de la muerte y el despliegue de la corrupción y la impunidad que los acompaña. Así mismo, pretenden evidenciar la condición cómplice de Estados que toleran la imbricada relación entre fuerzas criminales que actúan dentro y fuera de las instituciones o, para plantearlo de manera más directa, dentro de un imbricado colaboracionismo entre figuras institucionales, empresarios y miembros del crimen organizado (Valenzuela, 2015). Aunque esta situación no predomina en todos los casos revisados, es un panorama que evidencia cómo la violencia estatal y paraestatal —originada en las narrativas de la seguridad y la defensa del orden- legitima la estigmatización y la criminalización del sujeto joven.

\section{Acción conectiva, subjetividad y multimodalidad}

De acuerdo con Cabalín-Quijada (2014), la interacción de los jóvenes con y a través de las redes sociales es un fenómeno global; la juventud en Asia comparte experiencias 
similares a la de Estados Unidos y otras partes del mundo. ${ }^{6}$ Las tecnologías digitales y algunas herramientas de Internet (como las redes sociales, los blogs, las wikis y algunas aplicaciones de video y podcast, entre otras) sirven para desarrollar dos propósitos. Por un lado, publicar contenidos digitales sobre problemáticas que son denunciadas en los espacios virtuales, más allá de la información que divulgan los medios comerciales o institucionales, con el fin de producir resonancia ética y política en las audiencias, a modo de catarsis y concienciación, tal como ocurrió en los movimientos alteractivistas de 2011. Por otro lado, la producción, reproducción, circulación y recepción de textos multimodales que intensificaron los procesos de interacción y diálogo, promovieron prácticas de comunicación horizontales y reticulares, convocaron a otros participantes y articularon nuevos repertorios de acción tanto en el espacio digital como físico. Este último aspecto predominó en los estallidos sociales de 2019 .

Como se aprecia, en ambos hitos los medios digitales contribuyen a construir discursos compartidos de protesta y resistencia, así como proyectos alternativos que configuran progresivamente la acción conectiva juvenil. De acuerdo con Bennett y Segerberg (2011), este tipo de acción refiere a movilizaciones a gran escala, con escasos líderes, que adolecen de estructuras jerárquicas, las cuales se expanden a partir de prácticas comunicativas gestadas informalmente por ciudadanos identificados con la misma causa, sin controles ni filtros procedentes de estructura organizativa alguna. Estas prácticas comunicativas, generalmente desarrolladas por medio de memes, fotografías, videos, infografías, wikis, blogs y espacios en redes sociales, se gestionan a partir de un lenguaje común, un modelo de coautoría y de remix que se introduce en los espacios digitales, y que va dando forma a la inteligencia colectiva, en donde personas comunes y conectadas se vuelven protagonistas.

En los dos hitos de movilización examinados es posible apreciar cómo la lógica de la acción conectiva es también acción colectiva. El tipo de lógica que opera en los espacios digitales tiene como propósito no solo difundir información sino también influir, ocupar,

${ }^{6}$ Los usos de las tecnologías digitales y de las herramientas de Internet en el despliegue de los movimientos sociales, especialmente los de carácter juvenil y estudiantil, ha sido un tópico ampliamente trabajado por investigadores de las ciencias sociales, la comunicación y los estudios de juventud, durante los últimos diez años (Palenzuela, 2018). Al respecto, es posible distinguir dos grandes tendencias: en primer lugar, investigaciones que examinan los aspectos funcionales de las plataformas digitales, así como sus efectos sociales y políticos en la puesta en marcha de los movimientos (Cabalín-Quijada, 2014; Subirats, 2015); y, en segundo lugar, estudios que evidencian cómo se transforma la participación de los jóvenes en espacios no convencionales, así como los modos como estos hacen política y gestionan la acción colectiva en la esfera pública expandida (Antebi \& Sánchez, 2012; Scherman \& Arriagada, 2010). 
producir y transformar (Palenzuela, 2018). Estos propósitos se basan en el carácter performativo del lenguaje, situación que configura modos de acción conectiva de tipo simbólico-expresivo. De esta manera, los procedimientos y las estrategias que constituyen la acción conectiva también son llevados a cabo en los espacios físicos de la protesta. De acuerdo con Sampedro y Sánchez (2011, s. p.), quienes analizan el caso de los indignados del $15-\mathrm{M}$, la red era la plaza. Esto significa que determinadas prácticas propias de las redes, tales como, autoconvocarse, deliberar en foros, consumir contrainformación, así como operar en esferas públicas periféricas y digitales, se hicieron tangibles en las calles, las plazas y otros espacios de ocupación. Al respecto, tanto los alteractivistas de 2011 como los movimientos que agenciaron los estallidos sociales de 2019 evidencian que cada vez más se borra la frontera entre acción conectiva y colectiva, pues los atributos de la comunicación en los entornos digitales, especialmente la cooperación, la horizontalidad, la descentralización y la interconexión, se despliegan de manera cotidiana en las jornadas de protesta, las asambleas y las ocupaciones.

Si bien algunos autores han evidenciado el agotamiento de ciertos movimientos en sus prácticas presenciales e institucionales, luego de intensos procesos de acción conectiva, así como la existencia de barreras para que ciertas iniciativas transiten de lo digital a lo físico (Vissers \& Stolle, 2013), de acuerdo con Villanueva-Mansilla (2015) se puede afirmar que la base de estas dinámicas de participación juvenil radica en los grados de interacción alcanzados por los gestores de contenidos y las audiencias en torno a los problemas abordados. En el caso alteractivista de 2011, se evidencia que los debates producidos tanto en los medios sociales como en las calles se caracterizaron, no solo por los argumentos expuestos por los gestores de contenidos, sino también por la emergencia de aspectos emocionales en las audiencias que profundizaron sus vínculos con las acciones colectivas desplegadas tanto en lo virtual como en lo físico. Por su parte, en 2019 se observa que la intensificación de las interacciones de los activistas jóvenes (tal como lo revelan los movimientos de Hong Kong, Pakistán y Chile) hizo posible la ampliación de repertorios de acción, la profundización de prácticas de contienda y la capacidad de incidir a su favor en otros sectores y poblaciones.

Por otro lado, más allá del valor instrumental y estratégico de estos medios en las acciones conectivas/colectivas juveniles, es importante destacar cómo estas experiencias tecnomediadas no solo aportan a la redefinición de muchos aspectos relativos a la participación y la cultura política juvenil, sino también a la construcción de subjetividades. Al respecto, Bermúdez (2017) señala cómo las revueltas de 2011 en América Latina 
evidenciaron que muchos jóvenes, con historias de vida heterogéneas y sentidos de vida distintos, experimentaron formas de subjetivación basadas en posicionamientos éticos, políticos y culturales que pusieron en escena la intención de subvertir el orden hegemónico y propiciar una mejor calidad de vida social por medio de acciones de resistencia y nuevas ontologías relacionales (Escobar, 2005). Por su parte, Aguilar-Forero (2020) menciona cómo en los estallidos sociales de 2019 se evidencia una suerte de politización de la existencia tramitada por medio de lo digital, que permitió tanto en lo privado como en lo público desplegar otros modos de agrietar las estructuras que legitiman el abuso de poder y la criminalización del sujeto joven.

Otro aspecto que muestra los alcances de las acciones tecnomediadas en la construcción de la subjetividad juvenil son los aportes de los contenidos producidos por estos movimientos. Kress y van Leeuwen (2001) señalan que la multimodalidad comprende entramados de modos semióticos que componen los mensajes con el propósito de alcanzar aprendizajes y producir significados en el marco de situaciones comunicativas específicas. En la sociedad contemporánea, a partir de las dinámicas que subyacen a la convergencia mediática, semiótica y cultural (aspectos como la complementariedad, el ensamble y la hibridación de modos semióticos, asociados con los lenguajes de tipo gestual, kinestésico, visual, sonoro, audiovisual y digital), hacen posible no solo que los mensajes cuenten con un mayor potencial de recepción e interacción en las audiencias - al involucrar sus sentidos y emociones-, sino que también aportan a la construcción de otros regímenes de la mirada sobre los jóvenes y sus formas de lucha en la sociedad.

De este modo, se pudo evidenciar que, mientras los alteractivistas de 2011 desplegaron acciones conectivas por medio de información alimentada por usuarios (Facebook), apoyadas en la redifusión (microblogging) y basadas en contenidos producidos por audiencias (YouTube) (Villanueva-Mansilla, 2015), los movimientos de 2019, especialmente las prácticas políticas juveniles que se desarrollaron en Pakistán, Cataluña y Colombia, se caracterizaron por introducir contenidos hipermediales, multimediales y transmediales de carácter performativo a través de medios alternativos (Amador \& Muñoz, 2018) que, a modo de engagement, hicieron posible la generación de reacciones que fueron más allá de las pantallas, dado su potencial de esparcimiento en otras superficies digitales, sociales y físicas. 


\section{Conclusiones}

De acuerdo con las evidencias documentales de los dos hitos analizados, se pueden destacar tres tipos de conclusiones: primera, el papel de los jóvenes en el despliegue de los hechos de protesta y resistencia del movimiento alteractivista de 2011 y de los estallidos sociales de 2019, los cuales contienen aspectos comunes y diferencias; segunda, la relación constitutiva entre la acción colectiva y la condición juvenil contemporánea; y, tercera, las particularidades de la acción conectiva no solo como posibilidad de expansión de las formas de resistencia en la época de la convergencia mediática y cultural, sino también como práctica comunicativa, proceso de interacción y escenario para la emergencia de otros procesos de subjetivación de los sujetos jóvenes en los dos hitos problematizados.

En relación con la participación de los jóvenes en estas experiencias, se puede concluir que, tanto en los movimientos alteractivistas de 2011 como en los estallidos sociales de 2019, los jóvenes fueron protagonistas, y que los hechos que caracterizaron los dos hitos en cuestión evidencian que parte de la condición juvenil contemporánea está relacionada con la acción colectiva. Los datos muestran que una dimensión fundamental de la actual condición juvenil se constituye a partir de experiencias de re-existencia social y política que comprenden prácticas de protesta y resistencia, así como la generación de proyectos alternativos, en abierta disputa con los sectores hegemónicos, las instituciones y los procedimientos de la democracia formal.

De manera particular, los movimientos sociales juveniles antirracistas, feministas, ecologistas, en defensa de los derechos humanos, la memoria histórica, la diversidad sexual y de género, entre otros, que construyen permanentemente pluriversalidad, es decir, que abren espacio a multiplicidad de configuraciones político-culturales, diseños socioambientales y modelos económicos (Escobar, 2005), ponen en práctica un tipo de pensamiento relacional que se desprende de las prácticas políticas modernas (articuladas como derecha, centro e izquierda), abriéndose hacia otra cosa: la multiplicidad y la diferencia. En los dos hitos emerge un tipo de comportamiento que, en términos de Escobar (2005), no sigue necesariamente la lógica del orden, la centralización y la construcción jerárquica, sino la lógica de la diferencia, las redes, las singularidades espontáneas, la no-jerarquía, la nolinealidad, la autoorganización y, en últimas, el comportamiento adaptativo complejo.

Por otro lado, se observa que las acciones colectivas de 2011 están claramente identificadas con las luchas antiglobalización y antineoliberales. Parte de los movimientos 
alteractivistas (por ejemplo, Occupy Wall Street y las revueltas árabes) encontraron en los jóvenes y sus capacidades comunicativas, performativas y estéticas otras posibilidades para construir nuevos vínculos entre lo local y lo global. En este hito se presupone que las acciones colectivas antisistema tienen un potencial multiplicador y que la creación en red es factible desde una suerte de nuevo cosmopolitismo, el cual se apoya en el discurso de la justicia social, ambiental y cognitiva (Santos, 2010). Por su parte, en los estallidos sociales de 2019, la condición juvenil está atravesada por la precariedad, el desencanto y la violencia institucional, los cuales se evidencian en los cuerpos y los territorios que habitan los jóvenes. Estos elementos, los cuales se enmarcan en la noción de necropolítica, evidencian la centralidad del cuerpo en los procesos de tipo biopolítico, pero también en la acción colectiva desde la biorresistencia (Valenzuela, 2005). Esta refiere a diversas formas de biosignificación (biopoética, bioética), las cuales pueden actuar como tácticas de des-sujeción y transgresión corporal y significante, expresadas en la gestualidad, la estética, la lírica, la expresión artística, la performatividad y la resistencia cultural.

Por último, el análisis muestra que la acción conectiva y conectante, además de hacer posible la expansión de la acción colectiva, especialmente en el marco del llamado engagement, el cual refiere a la interacción e intercambio de información a través de los medios sociales en superficies amplias (virtuales o físicas) (Villanueva-Mansilla, 2015), se manifiesta de distintas maneras en estos dos movimientos. Por un lado, en los movimientos alteractivistas predomina un proceso de politización juvenil atravesado por la capacidad de incidencia en las audiencias por medio de la replicación y la redifusión de contenidos digitales. Por otro lado, en los movimientos de 2019 sobresale la intensificación de intercambios informacionales y la circulación recíproca de textos híbridos y remixados, entre productores de contenidos y audiencias, situación que contribuye a legitimar los objetivos de lucha, a fomentar la organización política de manera horizontal y reticular y a recrear la participación política juvenil desde abajo, sin permiso y en la diferencia.

\section{Referencias}

Achy, L. (2010). Substituer des emplois précaires à un chômage élevé. Les défis de lemploi au Maghreb. Carnegie Endowment for International Peace. https://bit.ly/zoUtUWl Aguilar-Forero, N. (2020). Las cuatro co de la acción colectiva juvenil: el caso del paro nacional de Colombia. Anal Político, 33(98), 26-43. https://doi.org/fnsb 
Aguilera, O. (2014). Generaciones: movimientos juveniles, políticas de la identidad y disputas por la visibilidad en el Chile neoliberal. Clacso.

Amador, J. C., \& Muñoz-González, G. (2018). Comunicación-Educación en Abya Yala: lo popular en la reconfiguración del campo. Nómadas, (49), 47-67. https://doi.org/ 10.30578/nomadas.n49a3

Amnistía Internacional. (2019, 16 de diciembre). Irán: miles de personas detenidas y en peligro de tortura en escalofriante represión después de protestas. http://bit.ly/3gQQEDD

Antebi, A., \& Sánchez, J. (2012). Plazas fuertes: de Midan Tahrir a la Plaça de Catalunya, espacio público y revueltas populares contemporáneas. En M. Urban-Crespo (Coord.), ¡Ocupemos el mundo! (pp. 67-84). Icaria Antrazyt.

Bellei, C., \& Cabalín, C. (2013). Chilean student movements: Sustained struggle to transform a market-oriented educational system. Current Issues in Comparative Education, $15(2), 108-123$.

Bennett, W. L., \& Segerberg, A. (2011) Digital media and the personalization of collective action: social technology and the organization of protests against the global economic crisis. Information, Communication \& Society, 14(6) 770-799. https://doi.org/ 10.1080/1369118x.2011.579141

Bermúdez, M. (2017). Subjetividades juveniles, expresiones políticas y uso de tecnologías digitales. Praxis y Saber, 8(17), 155-179. https://doi.org/fnsc

Cabalín-Quijada, C. (2014). Estudiantes conectados y movilizados: el uso de Facebook en las protestas estudiantiles en Chile. Revista Comunicar, 43, 25-33. https://doi.org/ 10.3916/C43-2014-02

Cárdenas, C. (2014). Inútiles y subversivos: representación transmedia de los estudiantes chilenos en redes sociales. Romanica Olomucensia, 26(2), 173-19o. https://doi.org/ $10.5507 /$ ro.2014.019

Castells, M. (2003). El poder de la identitat: l'era de la informació. UOC.

Cruz-Rodríguez, E. (2012). La Mane y el paro nacional universitario de 2011 en Colombia. Ciencia política, $7(14), 140-193$.

Chomsky, N. (2012). Ocupar Wall Street: indignados en el epicentro del capitalismo mundial. Tendencias.

Decenas de muertos y centenares de heridos en las protestas de Irak. (2020, 13 de febrero). RTVE. [Video]. http://bit.ly/37iffgB

Enríquez-Román, J. (2018). Dos experiencias americanas: Occupy Wall Street y Baltimore. Aposta. Revista de Ciencias Sociales, (80), 129-147. 
Escobar, A. (2005). Más allá del tercer mundo: globalización y diferencia. Instituto Colombiano de Antropología e Historia.

Fernández-Planells, A., Feixa, C., \& Figueras, M. (2013). 15-M En España: diferencias y similitudes en las prácticas comunicativas con los movimientos previos. Última Década, 21(39), 115-138. https://doi.org/10.4067/s0718-22362013000200006

Galeano, M. (2018). Estrategias de investigación social cualitativa: el giro de la mirada. Universidad de Antioquia. https://doi.org/10.2307/j.ctvdfo6h7

García, M. (2012). Una década de luchas estudiantiles. Cien días vistos por Cinep, (74), 17-20.

Hardt, M., \& Negri, A. (2004). Multitud. Paidós.

Ives, M., \& Li, K. (2019, 20 de junio). Para los jóvenes de Hong Kong, protestar es «una cuestión de vida o muerte». The New York Times. https://nyti.ms/34giZyj

Juris, J. (2012). Reflections on \#Occupy everywhere: Social media, public space, and emerging logics of aggregation. American Ethnologist, 39(2), 259-279. https://doi.org/ 10.1111/j.1548-1425.2012.01362.x

Juris, J., \& Razsa, M. (2012). Occupy, anthropology and the 2011 global uprisings. Cultural Anthropology, 27(2), 3-7.

Juris, J., Pereira, I., \& Feixa, C. (2012). La globalización alternativa y los «novísimos» movimientos sociales. Revista del Centro de Investigación de la Universidad La Salle, $10(37), 23-39$.

Kress, G., \& van Leeuwen, T. (2001). Multimodal discourse. Bloomsbury Academic.

Las cifras que dejó un año de estallido social en Chile. (2020, 17 de octubre). France 24. http://bit.ly/3alGTMu

Lazzarato, M. (2006) Por una política menor: acontecimiento y política en las sociedades de control. Traficantes de Sueños.

Manetto, F. (2019, 27 de noviembre). Las protestas en Colombia buscan redoblar la presión al Gobierno. El País. http://bit.ly/3alFfdM

Manetto, F., Oquendo, C., \&, Torrado, S. (2019, 4 de diciembre). Las protestas mantienen el pulso al Gobierno de Iván Duque en Colombia. El País. https://bit.ly/2Wexsqd

Martín, M. (2019, 28 de octubre). Jóvenes tras la hoguera. El País. http://bit.ly/34bDPPi

Mbembe, A. (2011). Necropolítica: seguido de Sobre el gobierno privado indirecto. Melusina.

Melucci, A. (1999). Acción colectiva, vida cotidiana y democracia. El Colegio de México. https://doi.org/10.2307/j.ctvhnoc2h

Morán, M. J., \& Benedicto, J. (2008). Las y los jóvenes como actores sociales y políticos en la sociedad global. Pensamiento Iberoamericano, (3), 139-164. 
Palenzuela, Y. (2018). Participación social, juventudes y redes sociales virtuales: rutas transitadas, rutas posibles. Última Década, 26(48), 3-34. https://doi.org/fnsd

Paúl, F. (2019, 23 de octubre). Protestas en Chile: 4 claves para entender la furia y estallido social en el país sudamericano. BBC News Mundo. http://bbc.in/37lamob

Paúl, F. (2019, 28 de noviembre). Protestas en Irán: por qué es tan difícil saber qué está pasando durante la ola de manifestaciones que ha dejado más de 140 muertos. BBC Mundo. http:// bbc.in/30TiIsX

Pérez-Beltrán, C. (2012). Las revueltas árabes de 2011: factores determinantes. Espacios Públicos, 15(33), 35-55.

Pleyers, G. (2018). Movimientos sociales en el siglo XXI: perspectivas y herramientas analíticas. Clacso. https://doi.org/10.2307/j.ctvnpokds

Protestas en Líbano: 4 claves para entender las marchas sin precedentes en el país asiático (y por qué se conocen como la revolución del WhatsApp). (2019, 23 de octubre). BBC News Mundo. http://bbc.in $/ 3 \mathrm{mlP}_{2} 63$

Rojas, C. (2019, 8 de septiembre). Tres protestas coincidieron en París y hay al menos 160 personas arrestadas. France 24. http://bit.ly/3af2 $\mathrm{S}_{7} \mathrm{O}$

Sajid, I. (2019, 7 de agosto). Protestas en Pakistán por las medidas tomadas por India en Cachemira. Anadolu Agency. http://bit.ly/3oPd3E8

Sampedro, V., \& Sánchez, J, (2011). La red era la plaza. En V. Sampedro (Coord.), Cibercampaña: Cauces y diques para la participación. Las elecciones generales de 2008 y su proyección tecnopolítica (pp. 237-240). Editorial Complutense.

Santos, B. (2010). Para descolonizar el occidente: más allá del pensamiento abismal. Clacso; Prometeo Libros.

Scherman, A., \& Arriagada, A. (2010). ¿Ciudadanía digital, participación tradicional?: jóvenes, participación política y consumo de medios en Chile [Ponencia]. III Congreso Latinoamericano de Opinión Pública, Querétaro, México.

Scherman, A., Correa, T., Peña y Lillo, Macarena, \& Pardo, J. (2020, 10 de enero). Encuesta jóvenes, participación y medios 2019. Vergara 240. http://bit.ly/3qXWzM3

Soengas-Pérez, X., \& Assif, M. (2017). El ciberactivismo en el proceso de cambio político y social en los países árabes. Comunicar, 53, 49-57. https://doi.org/10.3916/C53-2017-05

Subirats, J. (2015). Todo se mueve: acción colectiva, acción conectiva. Movimientos, partidos e instituciones. RES, (24), 123-131.

Tarrow, G. (2011). Power in movement. Cambridge University Press. 
Torres, Á. (2017, 3 de abril). La importancia de los idiomas de las publicaciones cientificas. Revista Comunicar, Escuela para autores. https://doi.org/10.3916/escuela-deautores-036

Valdettaro, S. (2012). Fuego-Revolución-Tecnologías. En M. Carlón, A. F. Neto (Eds.), La política de los internautas: nuevas formas de participación (pp. 155-172). La Crujía.

Valenzuela, J. M. (2005). El futuro ya fue: juventud, educación y cultura. Anales de la Educación Común, 1(1-2), 1-38.

Valenzuela, J. M. (Coord.) (2015). Juvenicidio: Ayotzinapa y las vidas precarias en América Latina y España. NED; Instituto Tecnológico y de Estudios Superiores de Occidente; El Colegio de la Frontera Norte.

Vasilachis, I. (2007). Estrategias de investigación cualitativa. Gedisa.

Villanueva-Mansilla, E. (2015). Acción conectiva, acción colectiva y medios digitales: posibilidades para la comunicación política en los tiempos de Internet. Contratexto, (24), 57-76. https://doi.org/10.26439/contratexto2015.no24.587

Vissers, S., \& Stolle, D. (2013). The Internet and new modes of political participation: Online versus offline participation. Information, Communication \& Society, ${ }_{17}(8)$, 937-955. https://doi.org/10.1080/1369118x.2013.867356

Zibechi, R. (2010). América Latina: contrainsurgencia y pobreza. Desde Abajo. 